\title{
Myocardial $T_{2}$ Mapping With Respiratory Navigator and Automatic Nonrigid Motion Correction
}

\author{
Shivraman Giri ${ }^{1,2}$, Saurabh Shah ${ }^{3}$, Hui Xue ${ }^{4}$, Yiu-Cho Chung ${ }^{5}$, Michael L. Pennell ${ }^{6}$, Jens \\ Guehring $^{4}$, Sven Zuehlsdorff ${ }^{3}$, Subha V. Raman ${ }^{2,7,8}$, and Orlando P. Simonetti ${ }^{1,2,7,8,{ }^{*}}$ \\ ${ }^{1}$ Department of Biomedical Engineering, The Ohio State University, Columbus, Ohio, USA. \\ ${ }^{2}$ Dorothy M. Davis Heart \& Lung Research Institute, The Ohio State University, Columbus, Ohio, \\ USA. \\ ${ }^{3}$ Siemens Healthcare, Chicago, Illinois, USA. \\ ${ }^{4}$ Siemens Corporate Research, Princeton, New Jersey, USA. \\ ${ }^{5}$ Shenzhen Institute of Advanced Technology, Chinese Academy of Sciences, Shenzhen, China. \\ ${ }^{6}$ Division of Biostatistics, College of Public Health, The Ohio State University, Columbus, Ohio, \\ USA.
}

${ }^{7}$ Department of Internal Medicine, Division of Cardiovascular Medicine, The Ohio State University, Columbus, Ohio, USA.

${ }^{8}$ Department of Radiology, The Ohio State University, Columbus, Ohio, USA.

\begin{abstract}
Quantitative $T_{2}$ mapping was recently shown to be superior to $T_{2}$-weighted imaging in detecting $T_{2}$ changes across myocardium. Pixel-wise $T_{2}$ mapping is sensitive to misregistration between the images used to generate the parameter map. In this study, utility of two motion-compensation strategies-(i) navigator gating with prospective slice correction and (ii) nonrigid registrationwas investigated for myocardial $T_{2}$ mapping in short axis and horizontal long axis views. Navigator gating provides respiratory motion compensation, whereas registration corrects for residual cardiac and respiratory motion between images; thus, the two strategies provided complementary functions. When these were combined, respiratory-motion-induced $T_{2}$ variability, as measured by both standard deviation and interquartile range, was comparable to that in breathhold $T_{2}$ maps. In normal subjects, this combined motion-compensation strategy increased the percentage of myocardium with $T_{2}$ measured to be within normal range from $60.1 \%$ to $92.2 \%$ in short axis and $62.3 \%$ to $92.7 \%$ in horizontal long axis. The new motion-compensated $T_{2}$ mapping technique, which combines navigator gating, prospective slice correction, and nonrigid registration to provide through-plane and in-plane motion correction, enables a method for fully automatic and robust free-breathing $T_{2}$ mapping.
\end{abstract}

\footnotetext{
"Correspondence to: Orlando P. Simonetti, Ph.D., The Ohio State University, 460 West 12th Avenue, Room 316 Biomedical Research Tower, Columbus, OH 43210. Orlando.Simonetti@ osumc.edu.
} 


\section{Keywords}

$T_{2}$ mapping; motion-compensation; nonrigid registration; respiratory navigator

Myocardial $T_{2}$ is altered in certain pathologies such as acute ischemia (1), myocarditis (2), and heart transplant rejection (3). Previous studies have proposed the use of $T_{2}$-weighted imaging or $T_{2}$ quantification (4-6) to detect these changes. Recently, we described a technique for $T_{2}$ mapping of the myocardium and demonstrated its ability to overcome some of the limitations of qualitative $T_{2}$-weighted imaging (7). Techniques for $T_{2}$ quantification generally require multiple $T_{2}$-weighted images, each acquired at a different echo or $T_{2}$-prep $\left(T_{2} \mathrm{P}\right)$ time; thereafter, $T_{2}$ values are computed by fitting an exponential decay curve across the multiple images at each pixel (7) or within desired regions of interest (ROIs) (8). This process of fitting a curve through points (pixel values or average ROI intensities) from different images renders $T_{2}$-quantification sensitive to misregistration of the images caused by breathing motion, inconsistent cardiac rhythm, or gross subject motion. The $T_{2}$ values of misregistered pixels and ROIs may be inaccurate, degrading the overall precision of myocardial $T_{2}$ estimation. Thus, it is important to ensure proper alignment of multiple-echo images prior to $T_{2}$ quantification.

Recently, a fast nonrigid registration algorithm (9) was successfully adapted to registering first-pass perfusion images (10). However, to the best of our knowledge, the use of registration in $T_{2}$ quantification has not yet been demonstrated.

In this work, we propose an enhancement of our previous $T_{2}$ mapping scheme: the new sequence includes a respiratory navigator to gate the acquisition based on diaphragm position and to prospectively correct for head-to-foot displacement; thereafter, any residual misregistration is corrected by a nonrigid registration algorithm optimized for myocardial $T_{2}$ mapping. We hypothesize that this combination of motion-correction strategies will enable free-breathing $T_{2}$ mapping with results comparable to breath-hold acquisitions. We tested this hypothesis in normal subjects by comparing three parameters: (i) pixel-wise standard deviation (SD) of myocardial $T_{2}\left(\sigma T_{2}\right)$ throughout the myocardium, (ii) interquartile range of $\mathrm{T} 2$ (IQR-T2), and (iii) percentage of myocardium with normal $T_{2}\left(\% T_{2 \text { Normal }}\right)$. These parameters were measured using three different acquisition schemes (breath-hold, freebreathing without navigator, and free-breathing with navigator) and two postprocessing modes (with and without nonrigid registration). The effect of the two motion compensation schemes on these parameters was first evaluated individually; thereafter, the proposed technique - free-breathing acquisition with navigator and registration — was compared with breath-hold acquisition. Results were evaluated for two slices in each subject: a midventricular short axis (SAX) in which the heart tends to demonstrate in-plane motion and the horizontal long axis (HLA) view in which breathing can induce through-plane motion of the heart.

\section{MATERIALS AND METHODS}

The basic $T_{2}$ mapping sequence has been previously described (7). Briefly, $T_{2}$ maps were generated using a monoexponential, log-transformed linear least-squares fit to signal 
intensities at each pixel of three T2 Prepared Steady-State Free Precession $\left(T_{2} \mathrm{P}-\mathrm{SSFP}\right)$ images (11). The $T_{2} \mathrm{P}$ times used were 0 (no prep), 24, and $55 \mathrm{~ms}$, and the ensuing SSFP readout was single-shot acquisition with linear $k$-space reordering. The nonzero $T_{2} \mathrm{P}$ times were achieved by a $T_{2} \mathrm{P}$ pulse consisting of a $90^{\circ}$ tip-down followed by four $180^{\circ}$ composite pulses with Malcolm-Levitt (MLEV) phase cycling scheme and a composite $90^{\circ}$ tip-up pulse; all the pulses were nonselective. Such a $T_{2} \mathrm{P}$ scheme has been shown to be robust against amplitude of static (polarizing) field and amplitude of (excitation) radiofrequency field inhomogeneities at $1.5 \mathrm{~T}(12)$.

Two new features were incorporated into this sequence: respiratory navigator and nonrigid motion correction. Figure 1a shows the new acquisition scheme; note that a navigator echo was acquired prior to each image, and $T_{2} \mathrm{P}$-SSFP images were acquired every $3 \mathrm{RR}$ intervals to allow sufficient $T_{1}$ recovery. Navigator echo tracking of diaphragm position was used to compensate for respiratory motion using a combined strategy of gating and prospective correction of slice position; for gating, an acceptance window of $8 \mathrm{~mm}$ was used, empirically chosen as a good balance between imaging efficiency and motion sensitivity. An empirical correction factor of 0.6 (13) was used to prospectively correct for residual displacement of the slice in the head-to-foot direction. Other imaging parameters are listed in Table 1.

A fast variational nonrigid registration algorithm (9) was applied to register the $T_{2} \mathrm{P}-\mathrm{SSFP}$ images in a pair-wise manner, that is, the $24-\mathrm{ms} T_{2} \mathrm{P}$ image was selected as the reference frame, and both 0 - and 55-ms images were registered to it. The registration algorithm could be considered as an extension of the classic optical flow method (14). In this framework, a dense deformation field is estimated as the solution to a calculus of variation problem, which is solved by performing a compositional update step corresponding to a transport equation. The regularization is added by low-pass filtering the gradient images which are in turn used as a velocity field to drive the transport equation. To accelerate the convergence and to avoid local minima, a multiscale image pyramid is created. We selected the local cross correlation as the image similarity measure, as its explicit derivative can be efficiently calculated, and it is general enough to cope with intensity fluctuation and imaging noise between two adjacent $T_{2} \mathrm{P}$ images. Figure $1 \mathrm{~b}$ summarizes the process.

The nonrigid registration of three $T_{2} \mathrm{P}-\mathrm{SSFP}$ images and the subsequent generation of $T_{2}$ maps were performed in a fully automatic manner and implemented as part of the image reconstruction software of the MR scanner (1.5 T MAGNETOM Avanto, Siemens Healthcare, Erlangen, Germany). The motion-corrected images and $T_{2}$ maps were typically available within $\sim 1 \mathrm{~s}$ after image acquisition.

\section{Phantom Study}

To ensure that the nonrigid registration algorithm did not bias the estimated $T_{2}$ values, the $T_{2}$ maps of a static two-compartment nickel-doped agar-phantom (True $T_{1} / T_{2}: 1099 / 49 \mathrm{~ms}$ and $1242 / 58 \mathrm{~ms}$ ) were acquired with and without the nonrigid registration algorithm. True $T_{1}$ and $T_{2}$ values of these phantoms were measured using spin echo sequences with and without inversion recovery preparation, respectively, with pulse repetition time $=10 \mathrm{~s}$ for each sequence (acquisition time per image was $\sim 17 \mathrm{~min}$ ). 


\section{Volunteer Study}

Ten healthy subjects consented to participate in this Health Insurance Portability and Accountability Act (HIPAA)-compliant study approved by the institution's Human Subjects Committee. For each subject, localizer scouts were first acquired to define mid-ventricular SAX and HLA slices. Thereafter, $T_{2} \mathrm{P}-\mathrm{SSFP}$ images were acquired under three conditions: (i) free-breathing without respiratory navigator, (ii) breath-hold (duration $=7$ RR intervals), and (iii) free-breathing with respiratory navigator. In each case, the acquisition was gated to mid-diastole, and $T_{2}$ maps were generated with and without nonrigid registration, yielding a total of six maps per slice.

\section{Image Analysis}

As the registration algorithm uses the center frame $\left(T_{2} \mathrm{P}=24 \mathrm{~ms}\right)$ as reference, this frame was unaffected by the algorithm and was the same in both unregistered and registered $T_{2} \mathrm{P}$ image sets; this image was selected to draw a ROI covering the entire myocardium in SAX view. For the HLA view, the apex was excluded from the ROI due to limited resolution and also to avoid partial volume effects. The ROI was then transferred to other images and to the $T_{2}$ maps for both unregistered and registered sets. These steps were performed using Matlab software (The Mathworks, Natick, MA).

First, mean $T_{2}$ values before and after registration were compared for any $T_{2}$ variation that may be caused as a side-effect of registration. Thereafter, the performance of motioncompensation strategies was evaluated with two performance metrics:

i. Variability of myocardial $T_{2}$, which was measured using $\mathrm{SD}\left(\sigma T_{2}\right)$ and interquartile range of $T_{2}$ (IQR-T $T_{2}$ values. While $\sigma T_{2}$ is a standard measure of dispersion, it is sensitive to extreme outliers; therefore, we include IQR- $T_{2}$, as it is less sensitive to outliers.

ii. Percentage of myocardial pixels with normal $T_{2}\left(\% T_{2 \mathrm{Normal}}\right)$, where normal $T_{2}$ was defined as the range of values within two SDs of mean $T_{2}$ in the breath-hold acquisition.

Additionally, pixels with abnormal $T_{2}$ values were investigated for connectivity, and a set of 10 or more connected abnormal pixels were considered to be a region of $T_{2}$ abnormality (15); given that the volunteers were healthy, all such cases represented false-positive diagnosis. The different motion-compensation strategies (registration, navigator, and navigator + registration) were compared with (i) free-breathing without compensation to demonstrate the improvement offered by the strategy and (ii) breath-hold acquisition (considered the gold-standard) to demonstrate the feasibility of using the strategy in a clinical setting.

\section{Statistical Analysis}

Myocardial $T_{2}$ values are expressed as mean $\pm \mathrm{SD}$. To test the hypothesis that nonrigid registration did not alter mean myocardial $T_{2}$ values, Student's $t$-test was used for the phantom and in vivo data. 
Signal intensities of misregistered pixels may not follow an exponential decay curve, or even a monotonically decreasing pattern; as a result, the fitted $T_{2}$ can vary over a wide range, including negative and very large positive values. The presence of such outlier pixels, along with other misregistered pixels, had two implications: (i) the high and low $T_{2}$ pixels could balance each other out, leaving the mean $T_{2}$ value unaltered; thus, we do not report the mean $T_{2}$ values and do not perform tests of significance for the mean $T_{2}$ values for $T_{2}$ maps generated from misregistered images. (ii) The resulting distributions of $\sigma T_{2}, \mathrm{IQR}-T_{2}$, and $\% T_{2 \text { Normal }}$ values across subjects were nongaussian; to account for this, $\sigma T_{2}$, IQR- $T_{2}$, and $\% T_{2 \text { Normal }}$ for different cases (combinations of acquisition and postprocessing) were compared using nonparametric Wilcoxon signed rank tests. A $P<0.05$ was deemed statistically significant, and Bonferroni correction was used for multiple comparisons.

All analyses were performed using MINITAB statistical software package (Minitab Inc., State College PA).

\section{RESULTS}

\section{Effect of Registration on $T_{2}$ Values}

Phantom results are shown in Table 2 and indicate that the image registration algorithm caused no change in $T_{2}$ values. For breath-hold acquisitions in the 10 subjects, the pixelwise $T_{2}$ values in SAX view were $52.3 \pm 6.5 \mathrm{~ms}$ before and $53.4 \pm 6.1 \mathrm{~ms}$ after registration, whereas for HLA view, the values were $54.5 \pm 7.1 \mathrm{~ms}$ and $56 \pm 6.8 \mathrm{~ms}$ ( $P$ value $=$ nonsignificant (NS) for both). These results show that the use of nonrigid registration did not have a significant side-effect on the distribution of myocardial $T_{2}$ values.

Results from individual and combined motion-compensation strategies are shown in Tables 3 and 4. In summary, registration alone reduced $\sigma T_{2}$ and IQR- $T_{2}$ and increased $\% T_{2 \text { Normal }}$ in all cases, giving $T_{2}$ maps that were comparable to those from breath-hold acquisitions. On the other hand, although navigator alone reduced $\sigma T_{2}$ and IQR- $T_{2}$ and increased $\% T_{2 \mathrm{Normal}}$, the resulting $T_{2}$ maps had higher variability of pixel-wise $T_{2}$ than those from breath-hold acquisitions; this was corrected for by combining navigator with registration, with the latter compensating for residual motion. Figures 3 and 4 demonstrate the benefit of adding registration to navigator acquisitions.

\section{Performance of Nonrigid Registration Only}

$T_{2}$ maps from free-breathing acquisitions compensated with only nonrigid registration were comparable to those from breath-hold acquisitions. Figure 2 demonstrates the effect of registration on $T_{2}$ values. In all 10 subjects, the proposed nonrigid registration significantly improved $(P<0.05) T_{2}$ maps by reducing pixel-wise $T_{2}$ variability as defined by (i) $\sigma T_{2}$ from 51.2 to $6.5 \mathrm{~ms}$ in SAX and 79.3 to $7.1 \mathrm{~ms}$ in HLA and (ii) IQR- $T_{2}$ from 25.8 to $6.5 \mathrm{~ms}$ for SAX and 26.1 to $10.1 \mathrm{~ms}$ for HLA, and increasing $\% T_{2 \text { Normal }}$ from 60.1 to $93.2 \%$ in SAX and 62.3 to $89.3 \%$ in HLA.

After registration, $T_{2}$ maps from free-breathing acquisition were comparable to those from breath-hold acquisition, with the median difference of $\sigma T_{2}=0.1 \mathrm{~ms} / 0.1 \mathrm{~ms}(P=\mathrm{NS}$ for both $)$, of IQR- $T_{2}=1.1 \mathrm{~ms}(P=0.08) / 1.6 \mathrm{~ms}(P=0.01)$, and that of $\% T_{2 \mathrm{Normal}}=1.9 \%(P=$ 
$0.48) / 5.7 \%(P=0.01)$ for SAX/HLA. Further, the residual outlier pixels in registered $T_{2}$ maps did not form a region of 10 or more connected pixels in any view (see column 2 in Figs. 3 and 4).

\section{Performance of Respiratory Navigator Only}

For the 20 acquisitions (two views in 10 subjects), navigator efficiency ranged from 15 to $100 \%$ (Median $=50 \% ; \mathrm{IQR}=67 \%$ ), resulting in a total scan time that ranged from a minimum of 7 to a maximum of $40 \mathrm{RR}$ intervals. The use of respiratory navigator alone improved $T_{2}$ maps in free-breathing acquisition; these maps were, however, inferior to those from breath-hold acquisitions.

Although navigator alone significantly improved $T_{2}$ maps $(P$ value $<0.05)$ by reducing pixel-wise $T_{2}$ variability as defined by (i) $\sigma T_{2}$ from 51.2 to $12.2 \mathrm{~ms}$ in SAX and 79.3 to 11.6 $\mathrm{ms}$ in HLA and (ii) IQR- $T_{2}$ from 25.8 to $9.4 \mathrm{~ms}$ for SAX and 26.1 to $9.9 \mathrm{~ms}$ for HLA and increasing $\% T_{2 \mathrm{Normal}}$ from 60.1 to $86.5 \%$ in SAX and 62.3 to $86 \%$ in HLA, Figs. 3 and 4 show volunteer images where navigator alone did not provide adequate motion compensation. Note also in Fig. 4 that the outlier pixels were connected, mimicking a region with $T_{2}$ abnormality; similar results were observed in $8 / 20$ other views.

Navigator alone gave $T_{2}$ maps that were inferior to those from breath-hold acquisition, with the median difference of $\sigma T_{2}=6.8 \mathrm{~ms}(P=0.05) / 5.2 \mathrm{~ms}(P=0.01)$, and that of $\% T_{2 \mathrm{Normal}}=$ $8.5 \% / 8.7 \%(P$ value $<0.05)$ for SAX/HLA. However, when expressed as IQR, the pixelwise $T_{2}$ variability was comparable to those from breath-hold acquisition; the median difference of IQR- $T_{2}$ was $1.7 \mathrm{~ms}$ for SAX and $1.8 \mathrm{~ms}$ for HLA $(P$ value $=\mathrm{NS})$.

\section{Performance of Navigator + Registration}

The benefit of additional compensation by registration over navigator gating alone is seen in column 3, bottom row of Figs. 3 and 4. Similar results were noted in other cases where navigator alone was inadequate.

For free-breathing $T_{2}$ mapping, the combined use of navigator and registration significantly improved $T_{2}$ maps ( $P$ value $<0.05$ ) by reducing pixel-wise $T_{2}$ variability as defined by (i) $\sigma T_{2}$ from 51.2 to $6.1 \mathrm{~ms}$ in SAX and HLA 79.3 to $6.6 \mathrm{~ms}$ in HLA and (ii) IQR- $T_{2}$ from 25.8 to $8.4 \mathrm{~ms}$ for SAX and 26.1 to $9.2 \mathrm{~ms}$ for HLA, and increasing $\% T_{2 \mathrm{Normal}}$ from 60.1 to $92.2 \%$ for SAX and 62.3 to $92.7 \%$ for HLA.

These $T_{2}$ maps were comparable to those from breath-hold acquisition, with median difference of $\sigma T_{2}=0.3 \mathrm{~ms} / 0.4 \mathrm{~ms}(P=\mathrm{NS})$, of IQR $-T_{2}=0.5 \mathrm{~ms} / 0.9 \mathrm{~ms}(P=\mathrm{NS})$, and of $\% T_{2 \text { Normal }}=2.8 \%(P=0.2) / 1.9 \%(P=0.04)$ for SAX/HLA. The residual outlier pixels did not form regions of ten or more connected pixels in SAX or HLA views in any of the volunteers.

\section{DISCUSSION}

This work has demonstrated that the combination of navigator gating and nonrigid motion compensation enables free-breathing myocardial $T_{2}$ mapping with results comparable to data 
acquisition during breath-hold. Further, the nonrigid registration and $T_{2}$ map calculation are fully automatic, and the entire process is accomplished inline in less than $1 \mathrm{~s}$, enabling this method to be easily applied in the clinical setting.

Although our study showed that nonrigid registration alone can successfully motion-correct the images acquired during free-breathing, we do not generally recommend this approach, especially for HLA view. Respiratory-induced cardiac displacement is predominantly in the superior-inferior direction, with the displacement in the other two orthogonal directions being relatively less (13); this translates to a greater through-plane motion for HLA and a greater in-plane motion for SAX in the majority of patients. Focal pathologies that are limited in spatial extent may not be captured in every 2D frame due to through-plane motion; indeed, Kellman et al. (16) reported this effect in multiple free-breathing single-shot late gadolinium enhancement HLA images but did not observe it in the SAX view. For $T_{2}$ mapping, if the different $T_{2} \mathrm{P}$ frames do not capture the pathology, then the subsequent registration and $T_{2}$ curve-fitting will lead to inaccuracies in $T_{2}$ quantification; this was not a problem in this study in either SAX or HLA view because of the homogeneous myocardium found in healthy volunteers. We included the case of free-breathing acquisition with only nonrigid registration to demonstrate the robustness of the registration algorithm.

In clinical applications, either breath-hold or respiratory navigator gating should be used for respiratory compensation to ensure a reproducible position of the heart between images. Breath-hold acquisitions are prone to diaphragm drift (13), and our results showed imperfect motion compensation with navigator gating and prospective slice correction. These inadequacies can be addressed by the additional motion compensation provided by nonrigid registration, which has shown robust performance in this study. Further, nonrigid registration can mitigate the effects of in-plane misregistrations caused by inconsistent cardiac rhythm or patient movement. Such misregistrations could not be experimentally controlled in vivo, and hence we chose to acquire free-breathing images without navigator to demonstrate the effectiveness of our nonrigid registration under extreme motion. In this way, the two motion-compensation strategies - respiratory navigator and nonrigid registration—provide complementary motion compensation.

While a breath-hold of $7 \mathrm{RR}$ is within the reach of most individuals, we frequently encounter cardiovascular patients with substantial cardiac motion possibly due to diaphragm drift, failed attempt at voluntary breath-hold, patient noncooperation, or beat-to-beat variability in cardiac rhythm. In such cases, although registration alone can correct for inplane motion, navigator gating must be used to compensate for through-plane motion. Furthermore, certain applications requiring high resolution $T_{2}$ maps may warrant a segmented rather than single- shot $k$-space acquisition. Only navigator gating can compensate for motion between $k$-space segments; registration algorithms that work in image space will not be effective in this situation.

Recently, a nonrigid registration algorithm was proposed for motion-corrected averaging of myocardial late gadolinium enhancement images acquired in free-breathing (17); the objective was to improve signal-to-noise ratio (SNR) and contrast-to-noise ratio (CNR) by averaging the registered images. The algorithm used by that study exploited B-splines to 
parameterize the motion field; the deformation between control points was interpolated using B-spline kernels. It should be noted that all the input images had similar signal and contrast levels; accordingly, mean square error was used as the similarity measure. Our nonrigid registration algorithm, on the other hand, solves a partial differential equation to get the pixel-wise deformation, using local cross-correlation as the similarity measure. Although both approaches rely on gradient descent optimization, with their respective performances being largely determined by the similarity measure (mean square error vs. local crosscorrelation), the partial differential equation-based method leads to so-called diffeomorphic deformation, which is a motion field free of folding or tearing; this diffeomorphic property is generally not a characteristic of the B-spline deformation, unless explicit constraints are added to avoid any possible foldings by limiting the magnitude of spatial deformation on the B-spline control points (18); this will lead to slower convergence as a side-effect. Given this, and our objective of registering multiple $T_{2} \mathrm{P}$ images, each with a different signal and contrast level, we chose to use the partial differential equation-based nonrigid registration algorithm.

The pixel-wise $\sigma T_{2}$ found in this study is higher than previously reported values (7), possibly because contours, drawn to cover the entire myocardium, included border pixels with partial-volume effects. Additionally, the misregistered pixels varied over a very wide range. In typical clinical studies, such pixels are excluded from image analysis by appropriate contouring. Given that the purpose of this study is to quantitatively demonstrate the effect of different motion-compensation strategies on $T_{2}$ maps, we did not exclude such pixels. We used two statistical measures of dispersion-SD and IQR - to demonstrate how motion correction and navigator gating effectively reduced motion-induced variability in myocardial $T_{2}$ values. While SD is a valid measure of dispersion even for nongaussian spread, it is sensitive to extreme outliers; accordingly, we utilized IQR as an additional measure of motion-induced $T_{2}$ variability that is less sensitive to the influence of outliers.

Several recent studies have demonstrated $T_{2}$ mapping with respiratory compensation. Foltz et al. (19) used respiratory bellows to monitor respiratory phase; this approach necessitated repetition of the acquisitions that were farthest from the median respiratory position. The authors of that study speculated that motion uncertainty was a primary contributor to remaining $\sigma T_{2}$ in vivo, suggesting inadequacy of their motion-compensation strategy. Huang et al. (20) used navigator gating for respiratory motion compensation and relied on a subjective analysis to either adjust ROI position or to discard images with more than 4 pixel misregistration. Blume et al. (21) also used navigator gating but did not account for the inplane misregistration that may arise due to patient movement or inconsistent cardiac rhythm. Compared to all previous approaches, the solution proposed in this paper is the only one to combine two techniques that provide complementary motion compensation (through-plane and in-plane). The nonrigid registration has shown robust performance even in the extreme case of free-breathing acquisition without navigator. Further, the navigator gating, nonrigid registration, and $T_{2}$ map generation are fully automatic and integrated with the sequence, obviating the need for any user input. 


\section{Limitations}

Given that the primary purpose of this study was to validate the nonrigid registration for $T_{2}$ mapping, patients were not recruited as the myocardial $T_{2}$ variability in patients would likely have confounded the investigation of variability due to misregistration. We did not investigate the effects of variable myocardial signal, as may be found in pathologies, on registration and subsequent $T_{2}$ mapping. We have recently demonstrated the feasibility of using breath-hold $T_{2}$ mapping with nonrigid motion correction (without navigator gating) in patients with acute myocardial infarction, myocarditis, and Tako-tsubo cardiomyopathy $(6,22)$.

While the sequence overestimated phantom $T_{2}$ values, which is consistent with and discussed in our earlier work (7), phantom results showed that registration did not affect $T_{2}$ quantification. Similarly, while the in vivo $T_{2}$ values postregistration did show a small systematic bias $(\sim+3 \%)$, it was not statistically significant. $\% T_{2 \mathrm{Normal}}$ in the proposed technique (navigator + registration) was less than the target $95 \%$ in many cases (Table 4); given that $\sigma T_{2}$ was similar to breath-hold acquisition, the reduced $\% T_{2 \text { Normal }}$ may be due to the aforementioned bias. In a quantitative approach, the resulting outlier pixels could potentially be flagged as pathologic tissue. A recent clinical study (15) defined a region as pathologic if 10 or more connected pixels had abnormal $T_{2}$-weighted signal. With this as a reference, the proposed technique (navigator + registration) performed well, because most of the outlier pixels were scattered around the borders, without forming a connected region (maximum eight connected pixels in this study), thus precluding potential false-positive cases (Fig. 4, column 3).

\section{CONCLUSIONS}

We have demonstrated a fully automatic and reliable free-breathing myocardial $T_{2}$ mapping technique based on single-shot, $T_{2}$-prepared SSFP that provides two complementary motioncompensation schemes: respiratory navigator and nonrigid registration. Both schemes were shown to independently provide significant motion compensation by reducing myocardial $T_{2}$ variability and increasing the percentage of myocardium with normal $T_{2}$ in healthy volunteers. While the navigator technique was used to ensure reproducible slice position, our results indicated that it alone did not provide adequate motion compensation for reliable $T_{2}$ mapping. A new nonrigid registration algorithm optimized for myocardial $T_{2}$ mapping was able to correct for any residual misregistrations; further, this algorithm alone showed robust performance even during breathing for both SAX and the motion-sensitive HLA views. When combined, these two strategies provide complementary motion compensation and can be used to generate fully automatic and reliable free-breathing myocardial $T_{2}$ maps.

\section{ACKNOWLEDGMENTS}

The content is solely the responsibility of the authors and does not necessarily represent the official views of the National Heart, Lung, and Blood Institute or the National Institutes of Health.

Grant sponsor: National Heart, Lung, and Blood Institute; Grant numbers: R01HL102450, R01HL095563. 


\section{REFERENCES}

1. Abdel-Aty H, Cocker M, Meek C, Tyberg JV, Friedrich MG. Edema as a very early marker for acute myocardial ischemia: a cardiovascular magnetic resonance study. J Am Coll Cardiol. 2009; 53:1194-1201. [PubMed: 19341860]

2. Friedrich MG, Sechtem U, Schulz-Menger J, Holmvang G, Alakija P, Cooper LT, White JA, AbdelAty H, Gutberlet M, Prasad S, Aletras A, Laissy JP, Paterson I, Filipchuk NG, Kumar A, Pauschinger M, Liu P. Cardiovascular magnetic resonance in myocarditis: A JACC White Paper. J Am Coll Cardiol. 2009; 53:1475-1487. [PubMed: 19389557]

3. Marie PY, Angioi M, Carteaux JP, Escanye JM, Mattei S, Tzvetanov K, Claudon O, Hassan N, Danchin N, Karcher G, Bertrand A, Walker PM, Villemot JP. Detection and prediction of acute heart transplant rejection with the myocardial $T_{2}$ determination provided by a blackblood magnetic resonance imaging sequence. J Am Coll Cardiol. 2001; 37:825-831. [PubMed: 11693758]

4. Abdel-Aty H, Zagrosek A, Schulz-Menger J, Taylor AJ, Messroghli D, Kumar A, Gross M, Dietz R, Friedrich MG. Delayed enhancement and $T_{2}$-weighted cardiovascular magnetic resonance imaging differentiate acute from chronic myocardial infarction. Circulation. 2004; 109:2411-2416. [PubMed: 15123531]

5. Aletras AH, Tilak GS, Natanzon A, Hsu LY, Gonzalez FM, Hoyt RF Jr, Arai AE. Retrospective determination of the area at risk for reperfused acute myocardial infarction with $T_{2}$-weighted cardiac magnetic resonance imaging: histopathological and displacement encoding with stimulated echoes (DENSE) functional validations. Circulation. 2006; 113:1865-1870. [PubMed: 16606793]

6. Verhaert D, Thavendiranathan P, Giri S, Mihai G, Rajagopalan S, Simonetti OP, Raman SV. Direct $T_{2}$ quantification of myocardial edema in acute ischemic injury. JACC Cardiovasc Imaging. 2011; 4:269-278. [PubMed: 21414575]

7. Giri S, Chung YC, Merchant A, Mihai G, Rajagopalan S, Raman SV, Simonetti OP. $T_{2}$ quantification for improved detection of myocardial edema. J Cardiovasc Magn Reson. 2009; 11:56. [PubMed: 20042111]

8. He T, Gatehouse PD, Anderson LJ, Tanner M, Keegan J, Pennell DJ, Firmin DN. Development of a novel optimized breathhold technique for myocardial $T_{2}$ measurement in thalassemia. J Magn Reson Imaging. 2006; 24:580-585. [PubMed: 16892203]

9. Chef d'hotel C, Hermosillo G, Faugeras O. Flows of diffeomorphisms for multimodal image registration. 2002 IEEE International Symposium on Biomedical Imaging, Proceedings. 2002:753756.

10. Xue H, Guehring J, Srinivasan L, Zuehlsdorff S, Saddi K, Chefdhotel C, Hajnal JV, Rueckert D. Evaluation of rigid and non-rigid motion compensation of cardiac perfusion MRI. Med Image Comput Comput Assist Interv Int Conf Med Image Comput Comput Assist Interv. 2008; 11(pt 2): $35-43$.

11. Kellman P, Aletras AH, Mancini C, McVeigh ER, Arai AE. $T_{2}$-prepared SSFP improves diagnostic confidence in edema imaging in acute myocardial infarction compared to turbo spin echo. Magn Reson Med. 2007; 57:891-897. [PubMed: 17457880]

12. Brittain JH, Hu BS, Wright GA, Meyer CH, Macovski A, Nishimura DG. Coronary angiography with magnetization-prepared $T_{2}$ contrast. Magn Reson Med. 1995; 33:689-696. [PubMed: 7596274]

13. Scott AD, Keegan J, Firmin DN. Motion in cardiovascular MR imaging. Radiology. 2009; 250:331-351. [PubMed: 19188310]

14. Xue H, Zuehlsdorff S, Kellman P, Arai A, Nielles-Vallespin S, Chefdhotel C, Lorenz CH, Guehring J. Unsupervised inline analysis of cardiac perfusion MRI. Med Image Comput Comput Assist Interv. 2009; 12(pt 2):741-749. [PubMed: 20426178]

15. Friedrich MG, Abdel-Aty H, Taylor A, Schulz-Menger J, Messroghli D, Dietz R. The salvaged area at risk in reperfused acute myocardial infarction as visualized by cardiovascular magnetic resonance. J Am Coll Cardiol. 2008; 51:1581-1587. [PubMed: 18420102]

16. Kellman P, Larson AC, Hsu LY, Chung YC, Simonetti OP, McVeigh ER, Arai AE. Motioncorrected free-breathing delayed enhancement imaging of myocardial infarction. Magn Reson Med. 2005; 53:194-200. [PubMed: 15690519] 
17. Ledesma-Carbayo MJ, Kellman P, Hsu LY, Arai AE, McVeigh ER. Motion corrected freebreathing delayed-enhancement imaging of myocardial infarction using nonrigid registration. $\mathrm{J}$ Magn Reson Imaging. 2007; 26:184-190. [PubMed: 17659545]

18. Rueckert D, Aljabar P, Heckemann RA, Hajnal JV, Hammers A. Diffeomorphic registration using B-splines. Med Image Comput Comput Assist Interv. 2006; 9(pt 2):702-709. [PubMed: 17354834]

19. Foltz WD, Al-Kwifi O, Sussman MS, Stainsby JA, Wright GA. Optimized spiral imaging for measurement of myocardial $T_{2}$ relaxation. Magn Reson Med. 2003; 49:1089-1097. [PubMed: 12768587]

20. Huang TY, Liu YJ, Stemmer A, Poncelet BP. $T_{2}$ measurement of the human myocardium using a $T_{2}$-prepared transient-state TrueFISP sequence. Magn Reson Med. 2007; 57:960-966. [PubMed: 17457877]

21. Blume U, Lockie T, Stehning C, Sinclair S, Uribe S, Razavi R, Schaeffter T. Interleaved T(1) and $\mathrm{T}(2)$ relaxation time mapping for cardiac applications. J Magn Reson Imaging. 2009; 29:480-487. [PubMed: 19161206]

22. Thavendiranathan P, Walls M, Giri S, Verhaert D, Rajagopalan S, Moore S, Simonetti OP, Raman SV. Improved detection of myocardial involvement in acute inflammatory cardiomyopathies using $T_{2}$ mapping. Circ Cardiovasc Imaging. 2011 Oct 28. Epub ahead of print. 

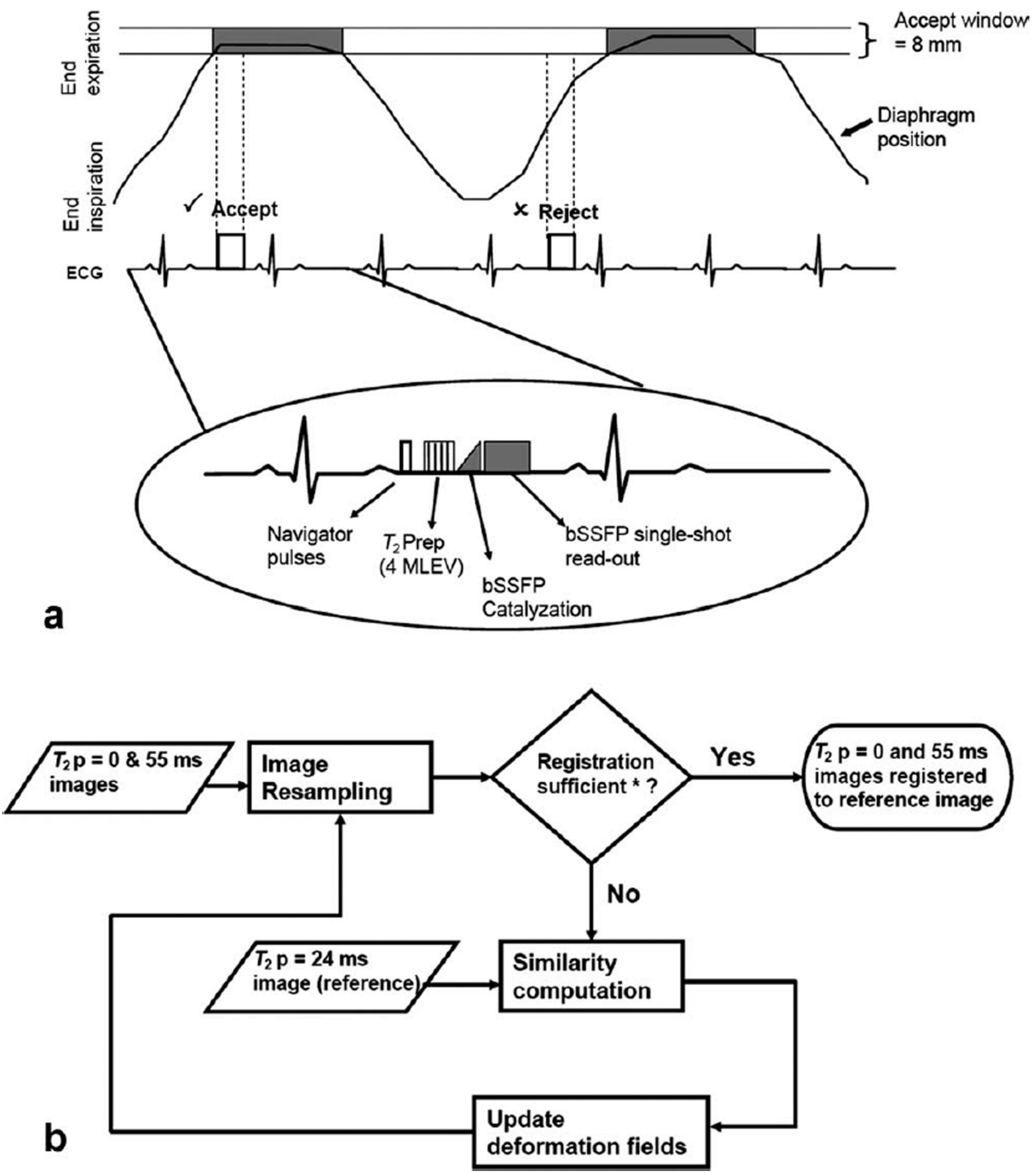

FIG. 1.

Acquisition scheme and postprocessing work-flow. a: Acquisition scheme for navigatorgated $T_{2}$ map using $T_{2} \mathrm{P}-\mathrm{bSSFP}$. Images were acquired once in every three cardiac cycles to allow for sufficient $T_{1}$ recovery between acquisitions. Acquired images were accepted only if the diaphragm position was within the acceptance window of $8 \mathrm{~mm}$ ("prospective correction of slice position" is not shown in the schematic). The sequence consists of navigator pulses, $T_{2} \mathrm{P}$ pulse (4 MLEV weighted), linear ramp catalyzation (for bSSFP) and single-shot bSSFP readout. b: Block diagram of motion correction using nonrigid 
registration. *"Registration sufficient" indicates that either the predefined similarity measure has been satisfied or the maximum number of iterations has been reached. 
$T_{2} \mathrm{P}=0 \mathrm{~ms}$

$T_{2} P=24 \mathrm{~ms}$

$T_{2} \mathrm{P}=55 \mathrm{~ms}$

$T_{2}$ Map
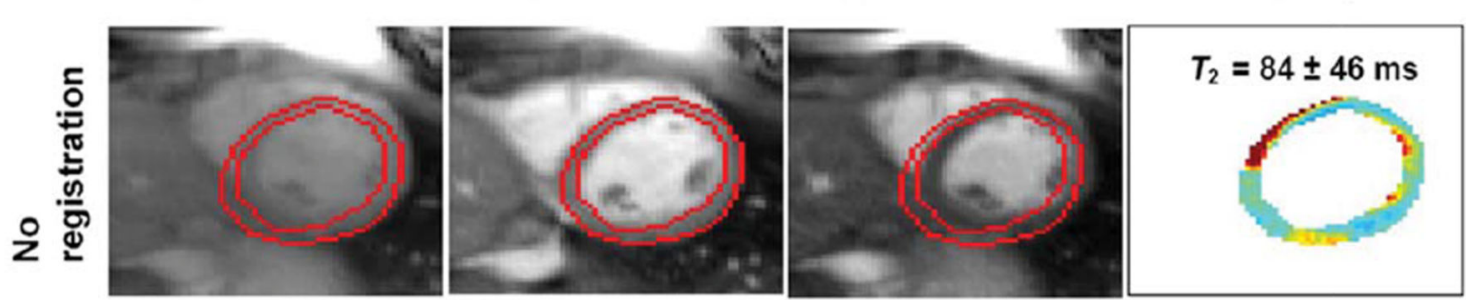

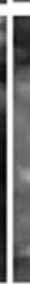
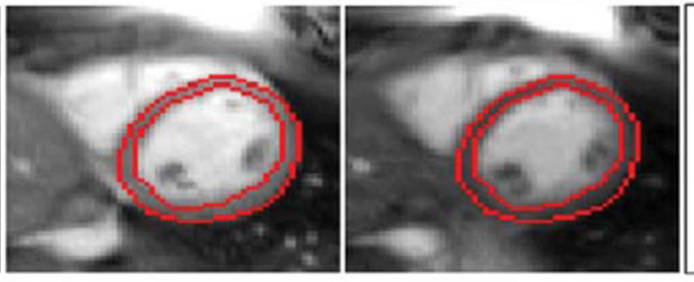

$T_{2}=55 \pm 6 \mathrm{~ms}$

a

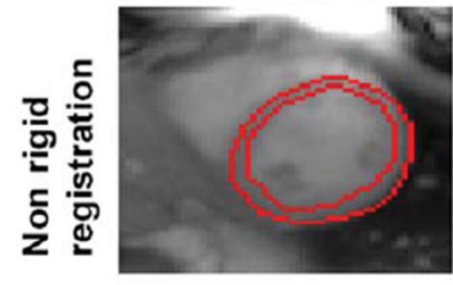

$T_{2} P=0 \mathrm{~ms}$

$T_{2} P=24 \mathrm{~ms}$

$T_{2} P=55 \mathrm{~ms}$

$T_{2}$ Map
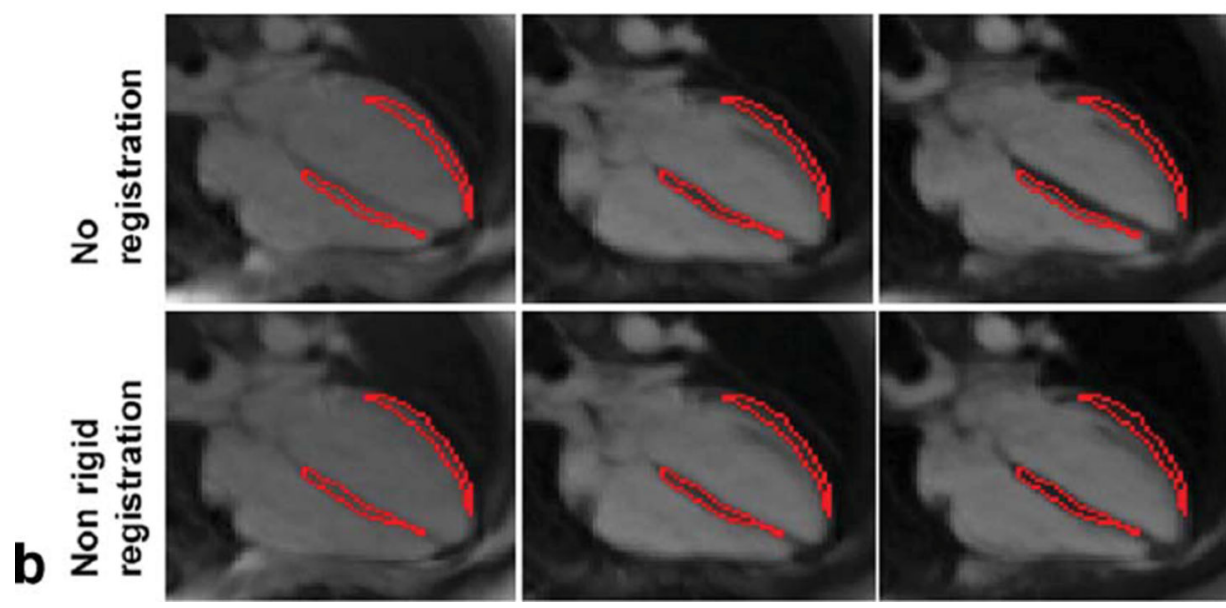

$T_{2}=78 \pm 37 \mathrm{~ms}$
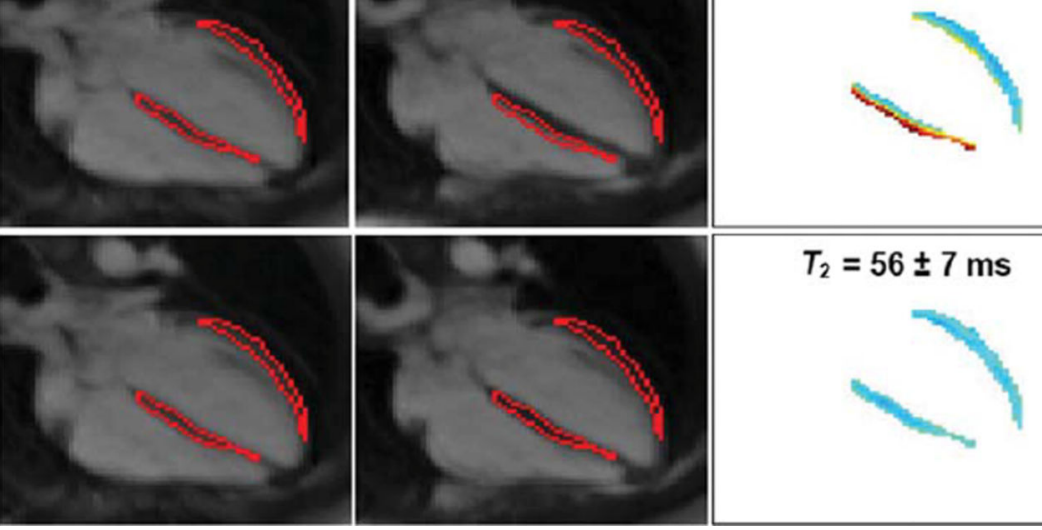

$T_{2}=56 \pm 7 \mathrm{~ms}$

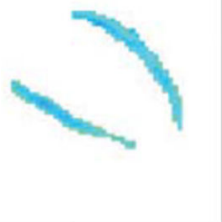

FIG. 2.

Effect of nonrigid registration on SAX (a) and HLA (b) $T_{2}$ maps of a healthy subject. Acquisition was free-breathing without respiratory navigator. For each view, $T_{2} \mathrm{P}-\mathrm{SSFP}$ images and $T_{2}$ maps preregistration (top row) and postregistration (bottom row) registration are shown. Contours were drawn on $T_{2} \mathrm{P}=24 \mathrm{~ms}$ image, the reference frame in registration. Myocardial $T_{2}$ map in the top row of each view contains misregistered pixels, resulting in heterogeneous $T_{2}$; after nonrigid registration, the myocardial $T_{2}$ is homogenous. Values expressed as mean \pm SD. Display window: 0-150. 

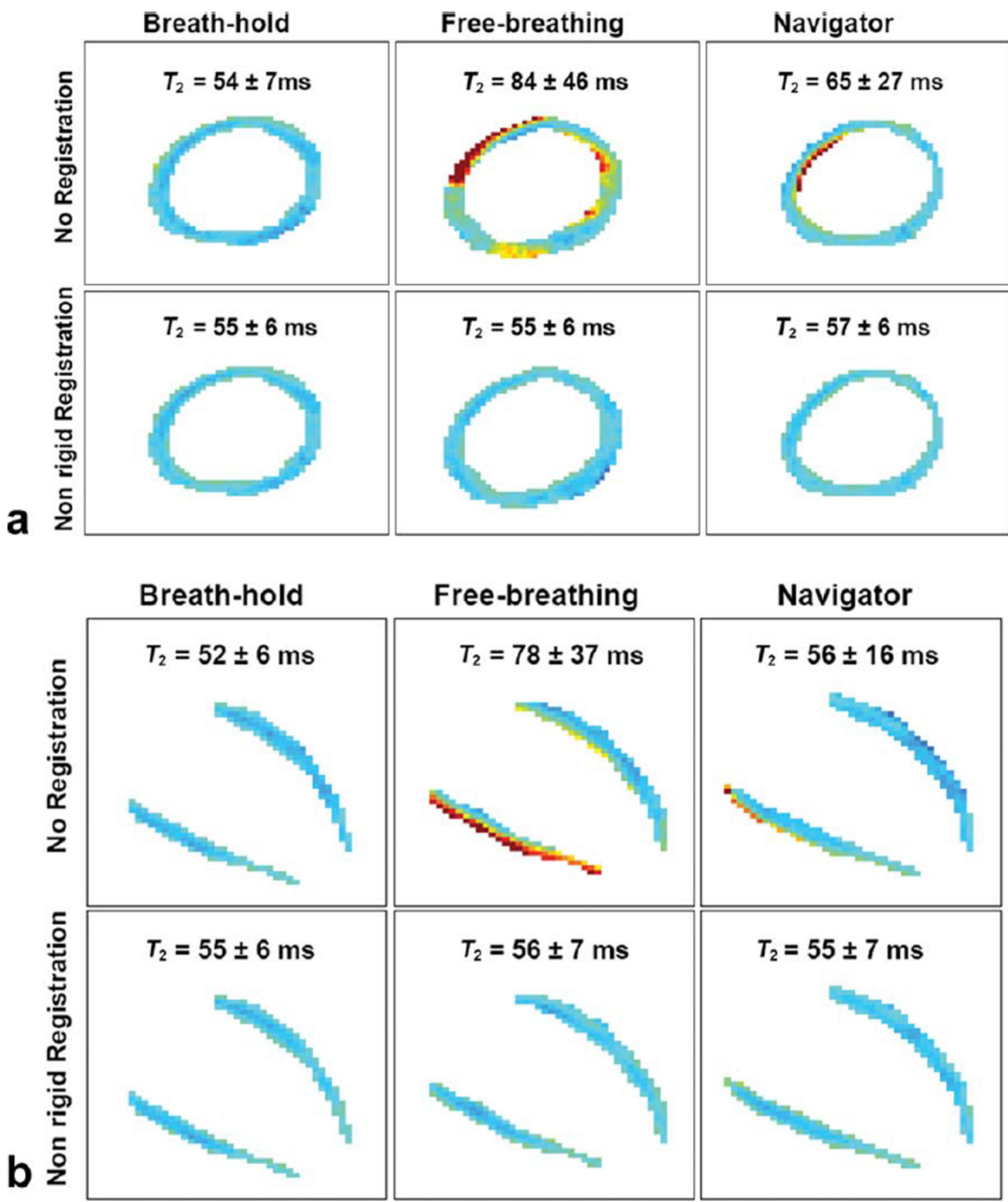

FIG. 3.

SAX (a) and HLA (b) $T_{2}$ maps from a healthy subject for each combination of acquisition and postprocessing. For each view, column 2 is free-breathing without navigator, and column 3 is free-breathing with navigator; nonrigid registration was used for bottom row only. Although the navigator reduced motion-induced $T_{2}$ variability (row 1, column 2 vs. column 3 for both views), residual $T_{2}$ heterogeneity was evident across the myocardium; registration improved $T_{2}$ map (row 2, column 3), yielding more homogeneous myocardial $T_{2}$ values. Also note that the nonrigid registration alone showed robust performance, correcting 
for motion-induced heterogeneity in $T_{2}$ values (column 2, row 1 vs. row 2). Values expressed as mean \pm SD. Display window: 0-150. [Color figure can be viewed in the online issue, which is available at wileyonlinelibrary.com.] 


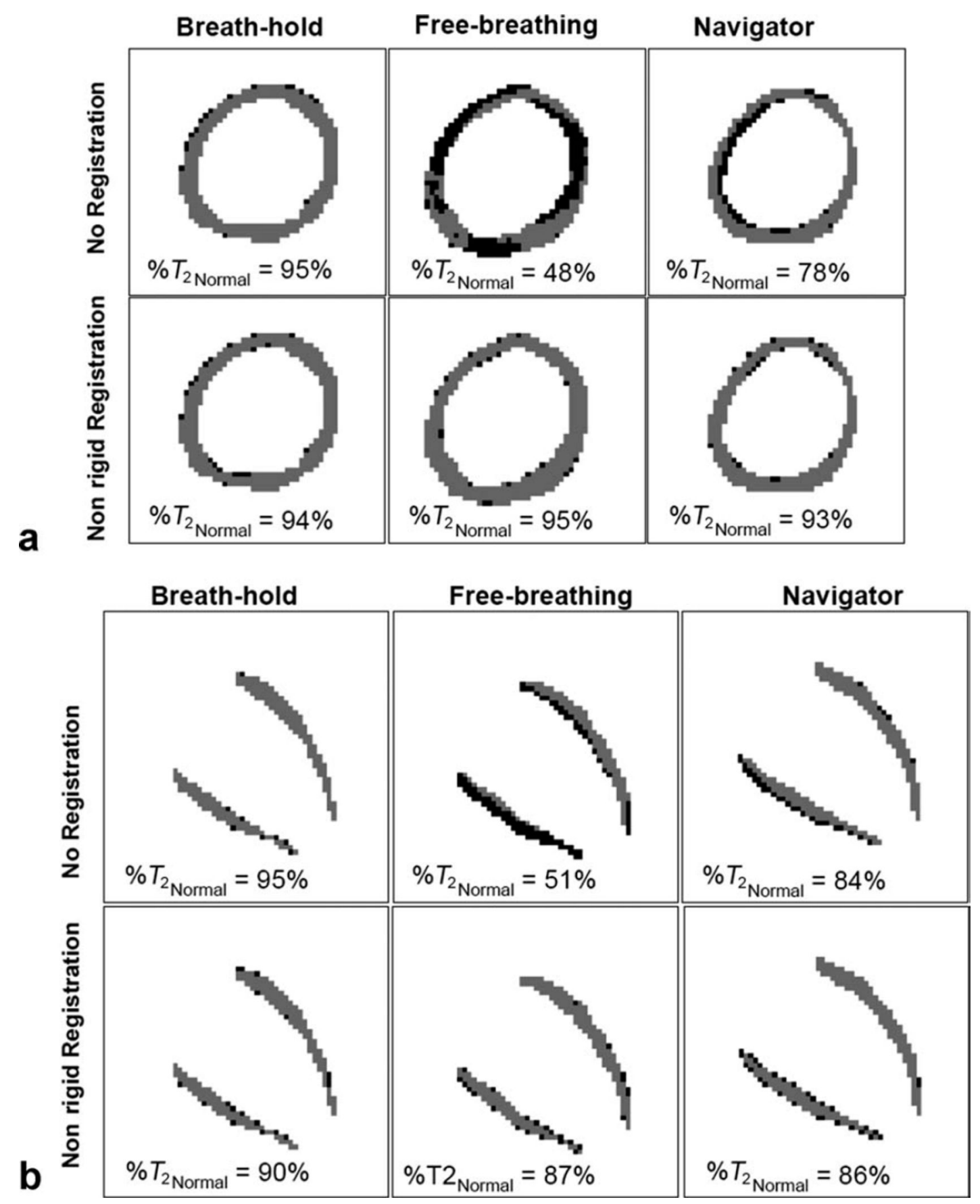

FIG. 4.

Pixels with normal (gray) and abnormal (black) $T_{2}$ values for different cases in the same subject shown in Fig. 3. Normal range of $T_{2}$ was defined in the breath-hold case (mean \pm $2 \mathrm{SD})$; for this case, the percentage of pixels with normal $T_{2}\left(\% T_{2 \mathrm{Normal}}\right)$ is $95 \%$ by definition. $\% T_{2 \text { Normal }}$ for other cases is shown in the images. Note that after registration, $\% T_{2 \text { Normal }}$ increases, with most of the outlier pixels scattered along the edges and not forming a cluster of connected pixels. 


\section{Table 1}

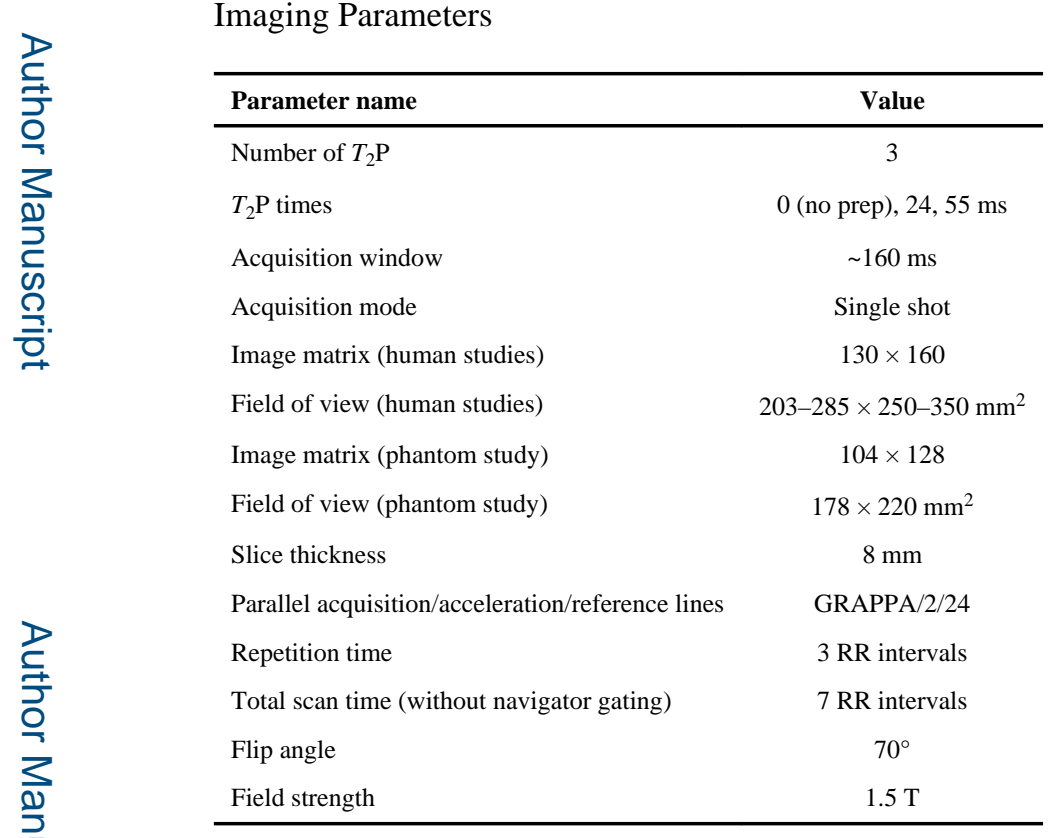


Table 2

Phantom Results Demonstrating the Effect of Nonrigid Registration on $T_{2}$ Values

\begin{tabular}{|c|c|c|}
\hline & Phantom 1 (True $T_{1} / T_{2}=1099 \mathrm{~ms} / 49 \mathrm{~ms}$ ) & Phantom 2 (True $T_{1} / T_{2}=1242 \mathrm{~ms} / 58 \mathrm{~ms}$ ) \\
\hline Without registration & $55.0 \mathrm{~ms} \pm 1.0 \mathrm{~ms}$ & $63.3 \mathrm{~ms} \pm 0.9 \mathrm{~ms}$ \\
\hline Without registration & $54.9 \mathrm{~ms} \pm 1.0 \mathrm{~ms}$ & $63.2 \mathrm{~ms} \pm 0.9 \mathrm{~ms}$ \\
\hline
\end{tabular}




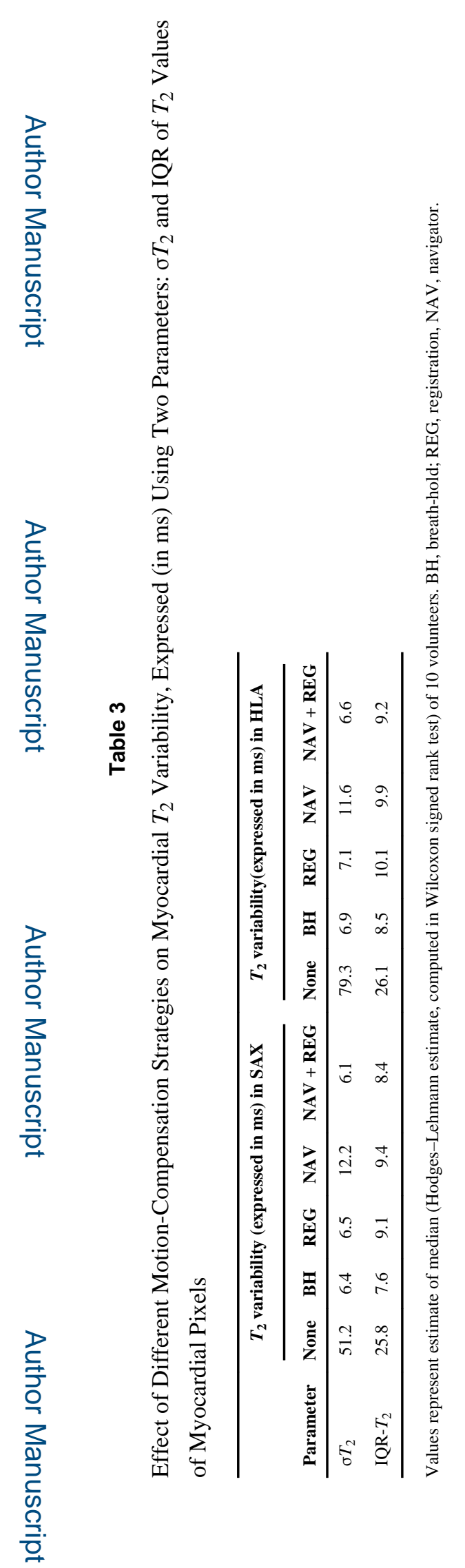

Magn Reson Med. Author manuscript; available in PMC 2015 July 23. 


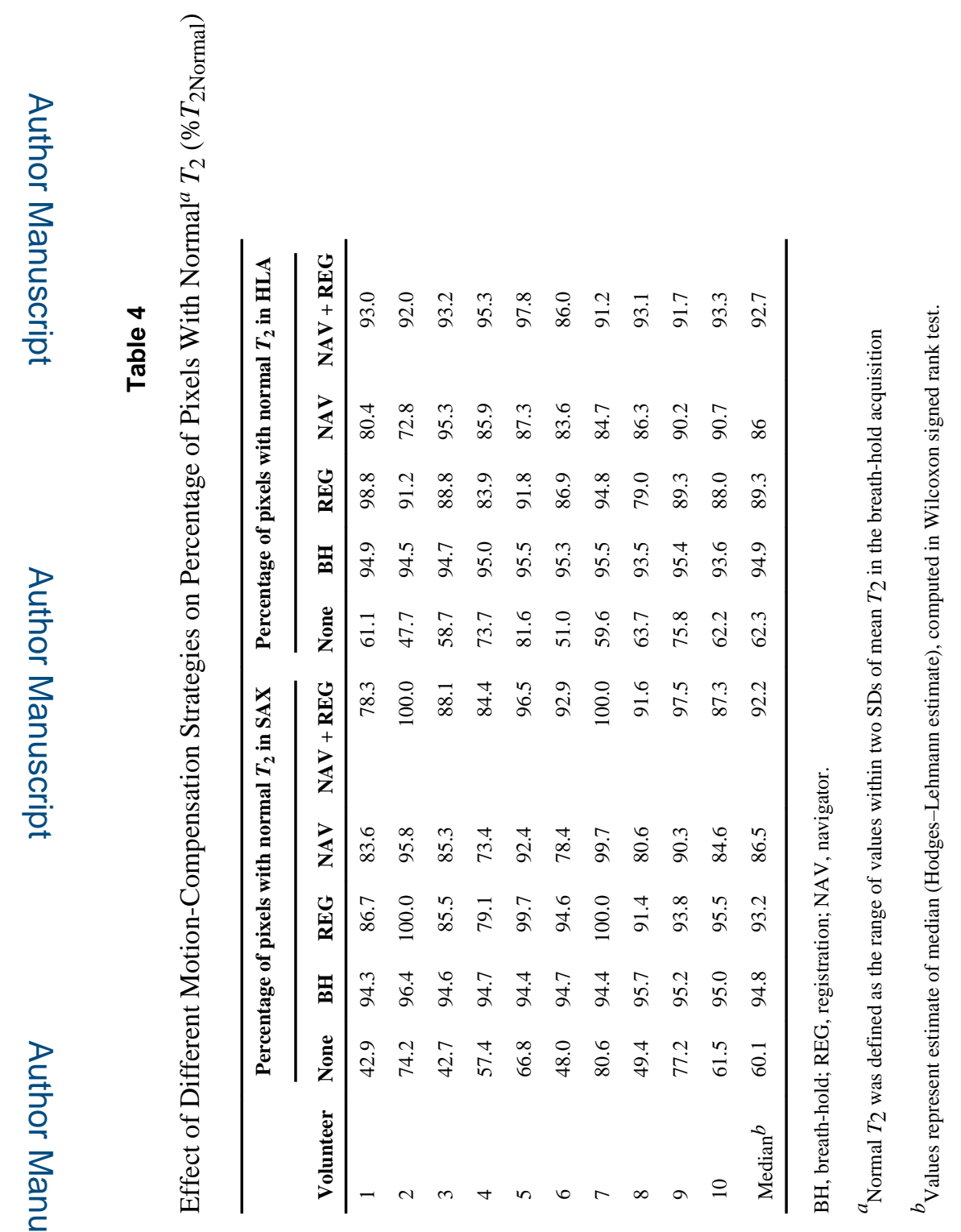

Magn Reson Med. Author manuscript; available in PMC 2015 July 23. 